\title{
Heartstart Scotland: the use of paramedic skills in out of hospital resuscitation
}

\author{
J N Adams, J Sirel, K Marsden, S M Cobbe
}

\begin{abstract}
Objective-To assess the frequency with which paramedic skills were used in out of hospital cardiac arrest and the effect of tracheal intubation on outcome.

Design-Retrospective analysis of ambulance service reports and hospital records. Setting-Scottish Ambulance Service and hospitals admitting acute patients throughout Scotland.

Results-A total of 8651 out of hospital resuscitation attempts were recorded and tracheal intubation was attempted in 3427 $(39.6 \%)$ arrests. One hundred and thirty six patients $(3.7 \%)$ were intubated and 476 $(9.1 \%)$ of the patients who were not intubated survived to hospital discharge $(p<0.001)$. Among the patients who were defibrillated the proportion intubated was highest in the patients who received the greatest number of shocks $(p<0.01)$. Among subjects receiving similar numbers of shocks survival rates were lower for intubated patients $(p<0.01)$. Patients with unwitnessed arrests were most frequently intubated and survival rates were lowest in this group.

Conclusions-Patients who are intubated seem to have lower survival rates. This may however reflect the difficulty of the resuscitation attempt rather than the effects of intubation. The use of basic life support skills rapidly after cardiac arrest is associated with the best survival rates. (Heart 1997;78:399-402)
\end{abstract}

Keywords: paramedics; resuscitation; myocardial infarction; tracheal intubation; prehospital care

Early defibrillation by a first responder has been shown to improve survival over basic life support alone. ${ }^{1}$ In recent years a number of projects has been established to provide and assess early defibrillation in out of hospital cardiac arrest in both Europe and the United States. ${ }^{2-4}$ Generally in these studies this service was provided by members of the fire service ${ }^{3}$ or medical or nursing staff ${ }^{2}$ manning a hospital based ambulance. In the United Kingdom, however, the first responders are the ambulance service. Before 1988 the Scottish Ambulance service had very few staff trained as paramedics and thus there was no scheme for prehospital defibrillation. The Heartstart Scotland programme started in 1988 with the aim of training all ambulance crews in defibrillation and equipped all 407 ambulances in Scotland with the Laederal 2000 automated external defibrillator (AED) by $1989 .{ }^{5}$ Since that time increasing numbers of paramedic ambulance personnel have been trained in advanced life support techniques including tracheal intubation and the administration of cardio-active medication. However the value of these skills used in the management of prehospital arrest has never been confirmed in a randomised clinical trial and it has been suggested that the use of paramedic skills results in a poorer outcome than defibrillation alone. ${ }^{6}$ This may reflect the condition of patients in whom paramedic skills are necessary, but could also be because of the use of the techniques themselves. The objective of this study was to establish the number of out of hospital arrests in which advanced life support skills were used by paramedics and the effect of these interventions on outcome.

\section{Method}

Since the start of the Heartstart programme all ambulance crews in Scotland summoned to a prehospital arrest where resuscitation is attempted have been instructed to complete a form recording details on patient identification, circumstances surrounding cardiac arrest, details of the resuscitation attempt, and the outcome. Further patient follow up was requested from the receiving hospital and all this information stored on a central database. The data collection form was modified in May 1991, according to the Utstein template ${ }^{7}$ and results since that time are presented. When analysing the data patients were categorised according to whether the arrest occurred after the arrival of the ambulance (crew witnessed), or before the arrival (bystander witnessed or unwitnessed). Statistical analysis was performed using a $\chi^{2}$ test and Mann-Whitney confidence intervals and test.

\section{Results}

From May 1991 to April 1994 a total of 8651 out of hospital resuscitation attempts was recorded. Over this period the number of resuscitation attempts increased from 2176 to 3209 per year and the number of patients intubated rose from $751(24.5 \%)$ to $1240(38.6 \%)$ per year. In total tracheal intubation was attempted in $3427(39.6 \%)$ cases and of these $136(3.7 \%)$ survived to hospital discharge compared with $476(9.1 \%)$ of the patients who were not intubated $(\mathrm{p}<0.001)$. Cardio-active drugs were used in 1887 arrests (21.4\%); adrenaline in $1208(14.0 \%)$ and atropine in $679(7.8 \%)$. A total of $561(6.5 \%)$ subjects had crew witnessed arrests, $5168(59.7 \%)$ were
Accepted for publication 19 June 1997 
Table 1 Number of resuscitation attempts and survival rates grouped according to number of DC shocks and endotracheal intubation

\begin{tabular}{llrc}
\hline $\begin{array}{l}\text { Number of } \\
\text { shocks }\end{array}$ & Intubated & $\begin{array}{l}\text { Number of } \\
\text { attempts }\end{array}$ & $\begin{array}{l}\text { Survived to } \\
\text { discharge } \\
(\%)\end{array}$ \\
\hline $1-3$ & Yes & $998(35.6)$ & $76(7.6)^{\star}$ \\
& No & $1805(64.4)$ & $350(19.3)$ \\
$4-6$ & Total & $2803(62.3)$ & $426(15.2)$ \\
& Yes & $473(45.9)$ & $33(7.0) \dagger$ \\
7 or more & No & $557(54.1)$ & $63(11.3)$ \\
& Yotal & $1030(22.9)$ & $96(9.3)$ \\
Total & No & $386(57.9)$ & $16(4.1) \ddagger$ \\
& Total & $281(42.1)$ & $30(10.6)$ \\
& & $667(14.8)$ & $46(6.9)$ \\
& & 4500 & $568(12.6)$ \\
\hline
\end{tabular}

${ }^{\star} \mathrm{p}<0.001 ; \mathrm{tp}<0.002 ; \neq \mathrm{p}<0.001$ for intubated $v$ non-intubated.

bystander witnessed, and 1855 (21.4\%) were unwitnessed. One hundred and fifty three $(27.2 \%)$ of the patients who had a crew witnessed arrest survived to discharge compared with $400(7.7 \%)$ and $21(1.1 \%)$ who sustained bystander and unwitnessed arrests, respectively $(\mathrm{p}<0.001)$. A total of 4151 $(47.9 \%)$ patients had electrocardiogram (ECG) tracings showing non-shockable rhythms and were therefore not defibrillated, 1570 (37.8\%) of these patients were intubated compared with $1857(41.2 \%)$ of the 4500 patients who received one or more DC shocks $(p<0.005)$. Some $1320(31.8 \%)$ of those with nonshockable rhythms had suffered unwitnessed arrests. Forty four $(1.1 \%)$ of the patients who were not defibrillated and $568(12.6 \%)$ of those who were defibrillated survived to hospital discharge $(p<0.001)$. Data from the subjects who received one or more DC shocks were reviewed separately and the results are shown below.

\section{NUMBER OF DC SHOCKS}

The number of patients and the percentage who survived to time of discharge fell significantly as the number of DC shocks given rose (table 1). The proportion of patients who were intubated was highest in the patients who received the greatest number of shocks $(\mathrm{p}<0.01)$. Survival rates in patients who were intubated were lower than for those who were not, but received a comparable number of shocks.

WITNESSED VERSUS UNWITNESSED

A total of $320(7.9 \%)$ subjects had crew witnessed, $3173(78.8 \%)$ bystander witnessed, and 535 (13.3\%) unwitnessed arrests (table 2). The proportion of patients intubated was greatest in the unwitnessed group. The percentage of patients intubated increased with increasing number of shocks in all three catagories and in each instance this was statistically significant. The percentage of patients surviving to hospital discharge decreased with increasing number of DC shocks in both the bystander witnessed and unwitnessed groups, in all three groups the survival rates were lower in the patients who were intubated. Multivariate regression analysis showed that both the number of shocks delivered and intubation were adverse predictors of the outcome of resuscitation, although there was no statistical interaction between the two.

\section{Discussion}

Several factors influence the outcome of out of hospital cardiac arrest. The early use of defibrillation, ${ }^{1}$ bystander cardiopulmonary resuscitation (CPR), ${ }^{8}$ and presentation with ventricular fibrillation ${ }^{9}$ have all been shown to improve survival. The Heartstart Scotland project provided automatic defibrillators to all ambulance units in Scotland ensuring that patients in ventricular fibrillation at the time of arrival of the ambulance could be defibrillated. ${ }^{10}$ Since the inception of this project ambulance personnel in increasing numbers have been trained in paramedic skills; to perform endotrachael intubation, venous cannulation, and to administer cardio-active medication. The advantage of using these skills over basic life support and defibrillation alone has not been established. Work by Guly et al suggests that the use of paramedic skills does not improve outcome. ${ }^{6}$ They showed that subjects in whom paramedic skills were used had similar presenting rhythms to those treated by basic life support and defibrillation and there was no difference in the proportion who sustained unwitnessed arrests or in whom bystander CPR was performed. The time at the scene of arrest was longer in the group in whom paramedic skills were used. Guly et al postulated that this was because of the use of these skills rather than the difficulty of the arrest, and that prolonged resuscitation at the scene resulted in poor survival. No mention was made of the number of shocks given to patients in ventricular fibrillation.

In this study, patients who presented with non-shockable rhythms (asystole, complete heart block, bradycardias, and electromechanical dissociation) had significantly lower survival

Table 2 Number of patients and survival rates according to number of shocks administered, presence of ambulance crew or witness for intubated and non-intubated subjects

\begin{tabular}{|c|c|c|c|c|c|c|c|c|c|}
\hline & \multirow[b]{2}{*}{ Intubated } & \multicolumn{2}{|l|}{ 1-3 shocks } & \multicolumn{2}{|l|}{ 4-6 shocks } & \multicolumn{2}{|l|}{$7+$ shocks } & \multicolumn{2}{|l|}{ Total } \\
\hline & & Number (\%) & Survived (\%) & Number (\%) & Survived (\%) & Number (\%) & Survived (\%) & Number (\%) & Survived (\%) \\
\hline \multirow[t]{3}{*}{ Crew } & Yes & $33(12.5)^{\star}$ & $4(12.1)$ & $9(26.5)^{\star}$ & $2(22.2)$ & $10(45.5)^{\star}$ & $0(0)$ & $52(16.3)$ & $6(11.5)$ \\
\hline & No & $231(87.5)$ & $125(54.1)$ & $25(73.5)$ & $9(36.0)$ & $12(54.5)$ & $7(58.3)$ & $268(84.7)$ & $141(52.6) \dagger$ \\
\hline & Total & 264 & $129(48.9)$ & 34 & $11(32.4)$ & 22 & $7(9.1)$ & 320 & $147(45.9)$ \\
\hline \multirow[t]{3}{*}{ Bystander } & Yes & $729(37.6)^{\star}$ & $59(8.9)$ & $270(38.5)^{\star}$ & $29(7.8)$ & $308(57.8)^{\star}$ & $13(4.2)$ & $1307(41.2)$ & $101(7.7)$ \\
\hline & No & $1210(62.4)$ & $199(16.4)$ & $431(61.5)$ & $52(12.0)$ & $225(42.2)$ & $9(8.4)$ & $1866(58.8)$ & $260(13.9) \dagger$ \\
\hline & Total & 1939 & $258(13.3)$ & 701 & $81(11.6)$ & 533 & $22(4.1)$ & 3173 & $361(11.4)$ \\
\hline \multirow[t]{3}{*}{ Unwitnessed } & Yes & $139(38.5)^{\star}$ & $3(2.0)$ & $61(50.0)^{\star}$ & $1(0.1)$ & $32(61.5)^{\star}$ & $0(0)$ & $232(43.4)$ & $4(1.7)$ \\
\hline & No & $222(61.5)$ & $13(5.8)$ & $61(50.0)$ & $0(0)$ & $20(38.5)$ & $0(0)$ & $303(56.6)$ & $13(4.3)$ \\
\hline & Total & 361 & $16(4.4)$ & 122 & $1(0.08)$ & 52 & $0(0)$ & 535 & $17(3.3)$ \\
\hline
\end{tabular}

${ }^{\star} \mathrm{p}<0.01$ for intubated patients with $1-3 v 4-6$ and $7+$ shocks. $\mathrm{tp}<0.01$ for intubated $v$ non-intubated. 
rates than those who presented with shockable rhythms (ventricular fibrillation or tachycardia). A slightly smaller proportion of the subjects with non-shockable rhythms were intubated compared with those who were defibrillated. Significantly more of the patients who presented with non-shockable rhythms had sustained unwitnessed arrests. As these arrests were unwitnessed we do not have accurate data on these subjects for the time between arrest and arrival of help, but it would seem reasonable to assume that in most of these cases the time elapsed would be longer than for witnessed arrests. It has been shown that even when basic life support is provided ventricular fibrillation frequently deteriorates to asystole if the patient is not defibrillated. ${ }^{11}$ Therefore it is unsurprising that a greater proportion of unwitnessed arrests presented with non-shockable rhythms. However the lower rate of intubation in patients with non-shockable rhythms is more difficult to explain. If a resuscitation is difficult and prolonged, as is often the case with patients in non- shockable rhythms, endotracheal intubation would usually be undertaken. Therefore it would be expected that the intubation rate would be higher in subjects with non-shockable rhythms. However if the condition of patients with non-shockable rhythms was generally poorer at the time of the arrival of the ambulance the resuscitation attempt might be less aggressive and intubation deemed futile.

The best survival rates were for patients who arrested with shockable rhythms after the arrival of the ambulance crew. In these patients arrest to DC shock intervals were very short and previous studies have shown quick response times improve survival. ${ }^{12}$ This group of patients had the lowest intubation rates and received fewer DC shocks. For both groups of patients with crew witnessed and bystander witnessed arrests, however, survival rates were significantly lower in subjects who were intubated. The proportion of patients intubated increased with the number of DC shocks given irrespective of whether the arrest was witnessed. The proportion of patients intubated was greatest in subjects who sustained unwitnessed arrests and received seven or more DC shocks. Survival rates were lowest in patients who received the most DC shocks, but when comparing subjects who received similar numbers of shocks patient survival rates were lower in patients who were intubated. Two possible explanations can be advanced to explain the poorer survival of intubated patients. The most plausible hypothesis is that the likelihood of survival is greatest in patients in whom the period of circulatory arrest and cerebral anoxia is at a minimum, and in whom defibrillation results in the rapid restoration of an effective cardiac output. Such patients are likely to recover consciousness rapidly and will not require intubation. As illustrated in table 2 the proportion of patients intubated increased with increasing numbers of shocks in each category of patient (crew witnessed, bystander witnessed or unwitnessed) consistent with the hypothesis that rapid restoration of a spontaneous circulation is associated with a lower requirement for intubation and that prolonged ventricular fibrillation predicts both an increased need for intubation and a poorer survival. The speed with which circulation is restored will depend on the rapidity with which basic life support and defibrillation are started, but also on the underlying cause of the cardiac arrest and other conditions from which the patient may be suffering.

The European Resuscitation Council guidelines call for intubation after three shocks if a spontaneous circulation has not been restored. Thus in theory all patients receiving four or more shocks should have been intubated. The reason that this was not the case in this series is that the ambulance staff attending the cardiac arrest may or may not have included a paramedic trained to intubate according to availability. In some cases a paramedic was called as a secondary responder to assist the ambulance technicians during a prolonged resuscitation attempt. Conversely according to resuscitation guidelines patients should only be intubated after three DC shocks have been given. In this series, however, over one third of the subjects receiving three or less shocks were intubated. In these cases the patient either regained a palpable pulse, but was not breathing spontaneously or having initially presented with a shockable rhythm developed an unshockable rhythm after receiving at least one DC shock. The association of intubation with a poorer outcome in such instances may be readily appreciated.

The alternative explanation for the association of intubation with poorer outcome is that the ambulance paramedics were either slow or inexpert in their intubation attempts and that the interruption of basic life support for attempts at intubation had an adverse effect on overall survival. Ambulance paramedics in Scotland are trained according to the standards of the Institute of Health and Care development and thus the implications of an adverse effect of intubation on survival would apply throughout the United Kingdom. Many factors influence the outcome of out of hosptial cardiac arrest and therefore only a randomised prospective trial of intubation versus conventional bag and mask management of the airway in cardiac arrest could resolve this issue definitively and it is unlikely that this will be performed.

The outcome of out of hospital cardiac arrest is best in patients who arrest in the presence of an ambulance crew, are defibrillated once, and do not require intubation. The association of poor outcome with intubation may reflect the patient's condition or the difficulties of prolonged resuscitation, but other factors cannot be ruled out and it is not possible to prove or refute the hypothesis that intubation in itself, in out of hospital arrest, results in a higher death rate.

1 Eisenberg MS, Coppas MK, Hallstrom AP, Blake B, Berger L, Short FA, et al. Treatment of out-of-hospital cardiac arrest with rapid defibrillation by emergency medical technicians. N Engl f Med 1980;302:1379-83.

2 Arntz HR, Oeff M, Willich SN, Storch WH, Schroder R. Establishment and results of an EMT-D program in a two- 
tiered physician escorted rescue system. The experience in Berlin, Germany. Resuscitation 1993;26:39-46.

3 Lombardi G Gallagher J Gennis P Outcome of out-ofhospital cardiac arrest in New York City. fAMA 1994;271 $678-83$

4 van der Hoeven JG, de Koning J, van der Weyden PK, Meinders AE. Improved outcome for patients with a cardiac arrest by supervision of the emergency medical services system. Neth f Med 1994;46:123-30.

5 Cobbe SM, Redmond MJ, Watson J, Hollingworth J, Carrington DJ. "Heartstart Scotland"-initial experience of a national scheme for out of hospital defibrillation. $B M \mathcal{F}$ 1991;302:1517-20.

6 Guly UM, Mitchell RG, Cook R, Steedman DJ, Robertson CE. Paramedics and technicians are equally successful at managing cardiac arrest outside hospital. BMF 1995;310: 1091-4.

7 Cummins RO, Chamberlain DA, Abramson NS, Allen M, Baskett PJ, Becker L, et al. Recommended guidelines for uniform reporting of out-of-hospital cardiac arrest: the Utstein style. Circulation 1991;84:960-75.
8 Herlitz J, Ekstrom L, Wennerblom B, Axelson A, Bang A, Holmberg S. Survival in patients found to have ventricular fibrillation after cardiac arrest witnessed outside hospital. Eur Heart f 1994;15:1628-33.

9 Herlitz J, Eckstrom L, Wennerblom B, Axelson A, Bang A, Holmberg S. Effect of bystander initiated cardiopulmonary resuscitation on ventricular fibrillation and survival after witnessed cardiac arrest outside hospital. Br Heart f 1994; 72:408-12. 10 Sedgwick ML, Dalziel K, Watson J, Carrington DJ, Cobbe
SM. Performance of an established system of first responder out-of-hospital defibrillation. The Heartstart Scotland Project in the 'Utstein Style'. Resuscitation 1993;26:75-88.

11 Enns J, Tweed WA, Donen N. Prehospital cardiac rhythm deterioration in a system providing only basic life support. Ann Emerg Med 1983;12:478-81.

12 van der Hoeven JG, Waanders $\mathrm{H}$, Compier EA, van der Wer Hoeven $\mathrm{JG}$, Waanders $\mathrm{H}$, Compier $\mathrm{EA}$, van der medical system. The prognosis in patients with an medical system. The prognosis in patients with an
out-of-hospital cardiac arrest. Neth $f$ Med 1994;44:5-11. 